\title{
Symptoms accompanying congenital ranula in neonates and infants - three case reports
}

\author{
Ewa Krasuska-Sławińska', Katarzyna Kurpiewska', Dorota Olczak-Kowalczyk², Dariusz Polnik², \\ Maciej Pronicki ${ }^{4}$ \\ 'Department of Paediatric Dental Surgery, The Children's Memorial Health Institute, Warsaw, Poland \\ ${ }^{2}$ Department of Paediatric Dentistry, Warsaw Medical University, Warsaw, Poland \\ ${ }^{3}$ Department of General Surgery, The Children's Memorial Health Institute, Warsaw, Poland \\ ${ }^{4}$ Department of Pathology, The Children's Memorial Health Institute, Warsaw, Poland
}

\section{ABSTRACT}

Congenital ranula is a rare lesion arising in the floor of the mouth, which may be asymptomatic and resolve spontaneously or can cause feeding and breathing difficulties in the child. It develops independently of systemic diseases. No diagnostic or treatment standards for congenital ranulas in neonates have been established so far. The paper describes clinical symptoms, diagnosis, and surgical management of congenital ranulas in three infants treated between 2015 and 2016 in the Children's Memorial Health Institute in Warsaw. Due to an abnormal body weight gain and difficulty in food intake overlapping with the presence of ranula, the patients were qualified for surgical treatment.

KEY WORDS:

congenital ranula, feeding difficulties, infant, neonate.

\section{INTRODUCTION}

Congenital ranula develops as a result of extravasation of saliva from salivary glands or ducts directly into adjacent oral floor tissues. This results from the fact that the sublingual salivary gland is unencapsulated [1-3]. Because of previously mentioned development mechanisms, in the literature ranula is often called a pseudocyst. A pseudocyst is a collection without a distinct membrane, as opposed to cyst, which is a sac with epithelium. In the rest of the article, we will use the terms ranula and pseudocyst interchangeably. In older children ranula most often arises from mechanical damage while chewing food. Some doctors believe that infants with congenital ranula may be associated with congenital duct malformation $[4,5]$. Small glands inside the salivary gland continuously produce mucus. Congenital or traumatic anomalies make it impossible for the salivary ducts to regenerate. A pseudocyst is formed as a result of mucus extravasation in the region of the sublingual gland [6]. It usually presents as a round, painless growth located in the bottom of the oral cavity. It turns bluish, which is related to a physical phenomenon known as the Tyndall effect (the light passing through the colloidal solution forms a light cone) [7]. Depending on the size and location, a congenital ranula may induce no complications or cause displacement of the tongue - from its slight elevation to protrusion out of the mouth, which prevents the child from bringing the upper and the lower lip together $[8,9]$. A child with congenital ranula is likely to avoid eating, and thus wake in the night, feed less frequently, suck the breast ineffectively, and spit up the milk. This leads to lack of weight

\section{ADDRESS FOR CORRESPONDENCE:}

Katarzyna Kurpiewska, Department of Paediatric Dental Surgery, The Children's Memorial Health Institute,

20 Dzieci Polskich Ave., 04-730 Warsaw, Poland, ORCID: 0000-0003-4278-1709, e-mail: kurpiewska.k@gmail.com 
gain and apathy in the child [10]. Congenital ranula is much less common. Some authors have estimated incidence of congenital ranula at $0.74 \%$ [4]. Other authors believe that the incidence is difficult to estimate due to the sparsity of cases described in the literature [11]. Its presence may not only cause feeding difficulties, but it can even lead to respiratory tract obstruction. For very large congenital ranulas, reported to reach diameters of up to $5 \mathrm{~cm}$, the airway obstructions may already occur at the prenatal stage, resulting in potential respiratory failure or e.g. aspiration of fluid from the ranula into the respiratory tract [12]. Because there are few publications in the literature concerning congenital ranulas, and the possibility of presenting three cases, we found this topic interesting.

\section{CASE 1 (KB)}

A male neonate born via C-section with a low birth weight of $2600 \mathrm{~g}$. Additional history of neonatal jaundice, congenital malformation of the fingers of both hands (oligodactyly), and facial dysmorphism. Feeding problems were reported by the mother since birth. The boy had difficulty sucking the breast and spat up some of the milk. When the boy was five months old, a growth was found on the floor of his mouth. Intraoral examination revealed a soft elastic growth $(0.5 \times 0.5 \mathrm{~cm})$ in the left-sided bottom of the oral cavity. Due to its small size, a decision
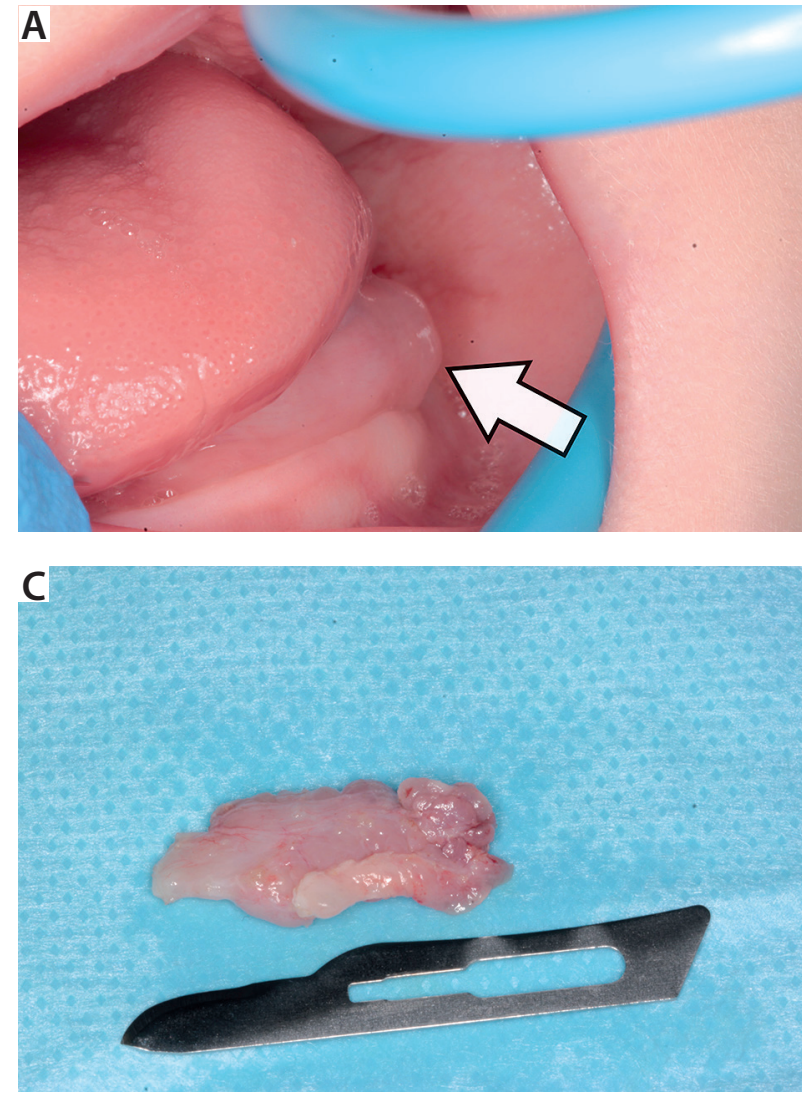

was made to postpone surgical intervention and follow up the patient. The patient was reported back after a month. The pseudocyst increased in size, slightly elevating the tongue $(2 \times 1 \mathrm{~cm})$, as shown in Figure 1A. When the patient turned 12 weeks, a decision was made to remove the ranula along with the sublingual gland $(2.5 \times 1 \times 0.5 \mathrm{~cm})$ under general anaesthesia. The post-excision status of the oral cavity and the removed tissue are shown in Figure 1B and 1C. The collected material was sent for histopathological examination, which showed a dilated central portion of the salivary duct with no signs of inflammation (Fig. 1D). The postoperative period was uneventful. The patient was discharged home on day three after the procedure. The boy was reported for follow-up visits after seven days, a month, and three months. He was monitored in the Children's Memorial Health Institute in Warsaw until the age of six months. Due to proper development, further care is provided by a paediatrician.

\section{CASE 2 (CI)}

A six-week-old female infant born at term via C-section with normal birth weight (3600 g) but with tuberous sclerosis complex (cardiac and CNS involvement). The patient gained no weight, and her feeding time was significantly prolonged. The child was lethargic, which raised parental concerns. A growth with an appearance corre-
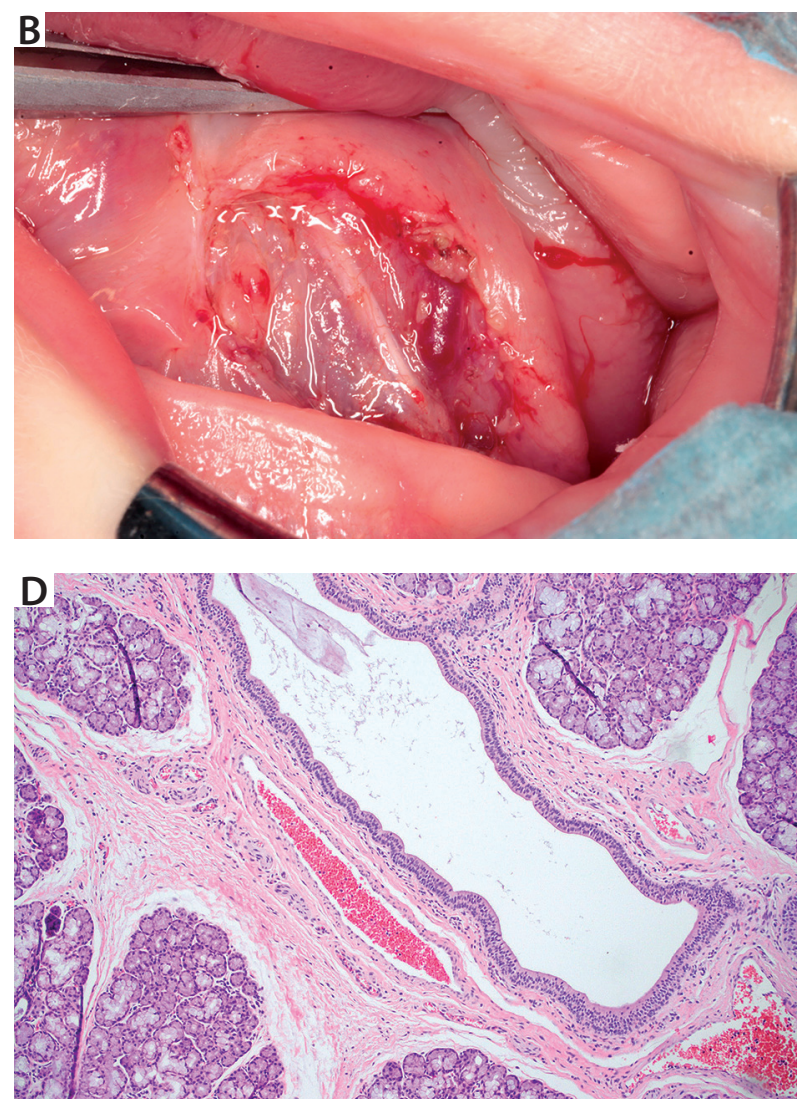

FIGURE 1. A) Clinical picture of the patient's oral cavity. The arrow indicates lesions in patient 1 (KB). B) Surgical field after ranula excision in patient 1 (KB). C) Enucleated salivary gland size. D) A 10× microscopic image - patient 1 
sponding to that of ranula was found at the left-side bottom of the oral cavity. After a one-month follow-up, at which point the lesion reached a size of $2 \times 1 \mathrm{~cm}$, a decision was made to perform a surgery. The six-week-old infant underwent a procedure to remove the ranula along with the left sublingual gland under general anaesthesia, using a traditional surgical approach. Because the child was feeling well, she was discharged home on day 3 after the procedure. Follow-up after two weeks and after a month confirmed a proper, uncomplicated healing process. Further care was provided by a primary care practitioner.

\section{CASE 3 (GB)}

A one-month-old boy born vaginally with normal birth weight (3340 g) but with congenital hydronephrosis. The child had difficulty feeding from birth, which led to the lack of weight gain. Clinical examination revealed bilateral, elastic growths at the bottom of the oral cavity (each about $1 \times 0.5 \mathrm{~cm}$ in size), as shown in Figure 2A, with more visible ranula on the right. The observed growth corresponded to the image of the ranula. The child underwent a surgery to remove the cystically distended left and right sublingual gland at the age of seven weeks. Surgical excision was performed under general anaesthesia. As a result of the procedure, the histopathological preparation of the sublingual gland was extracted. Figure $2 \mathrm{~B}$ shows a histopathological specimen of a distended sublingual gland.

Due to postoperative submandibular oedema, which was more pronounced on the left side, GB required further hospitalisation in the Intensive Care Unit and antioedematous treatment using Dexaven and furosemide for five days. He was discharged home 10 days after the surgery, but another hospital stay was needed after 23 days due to bilateral parotid enlargement. Ultrasound showed bilateral enlargement of parotid glands with abnormal echostructure, inhomogeneous hyperechogenicity, and multiple regional lymph nodes. Doppler ultrasound of the neck showed normal arterial and venous blood flow in the neck; no laboratory abnormalities were found. The diagnosis was extended to include immune testing. Because the oedema gradually resolved, a decision was made to discharge the child home and perform an elective MRI. The MRI, which was performed 1.5 months after the surgery, showed oedematous submandibular soft tissue, more pronounced on the left side, with no abscess or focal lesions. Submandibular and parotid glands had normal structure; no cervical lymphadenopathy was observed. Follow-up visits in the following weeks showed a gradual resolution of submandibular and peripharyngeal oedema, with complete resolution after three months.

Despite excision of congenital ranula, the child still presented with eating problems and abnormal development. Cytogenetic testing performed in the second half of 2017 revealed a rare disorder known as Nijmegen breakage syndrome.

\section{DISCUSSION}

Clinically, congenital ranula usually presents as a non-pulsatile translucent, blue, non-pedunculated, ovalto-round swelling with smooth surface. On palpation, the lesion is soft, smooth, non-tender, and non-bleeding. It shows slow growth [13]. The ranula is usually unilateral. Two of the described patients presented with left-sided ranulas, and one patient developed bilateral ranulas. The diagnosis of ranula is primarily based on clinical examination. However, differential diagnosis against a congestive cyst of the lower lip, lymphangioma, vascular malformation, haemangioma, middle cyst of the neck, and epidermoid cyst of the head and neck is always required [13-15].

Ultrasonography, computed tomography, and magnetic resonance imaging are used in the diagnosis. These tools are invaluable in determining the size and location of ranulae. According to Baker, the latter modality is the gold standard. High-resolution images allow for the differentiation of salivary duct and gastrointestinal abnormalities [16]. Fine-needle biopsy followed by biochem-
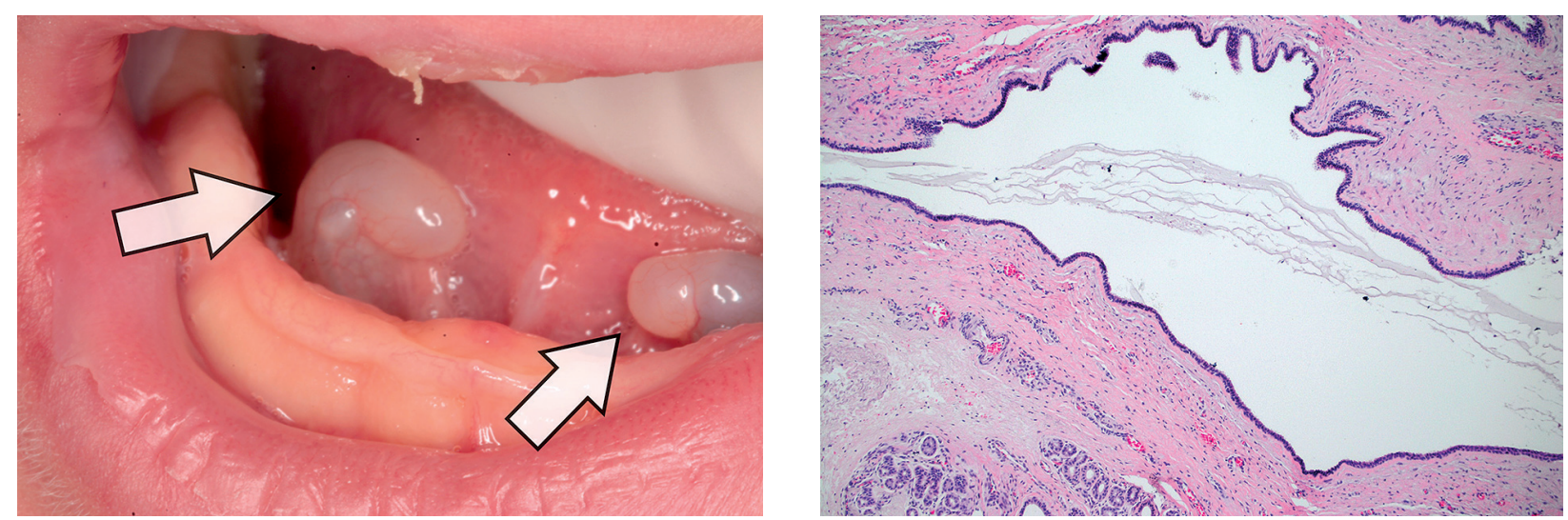

FIGURE 2. A) Clinical picture in patient 3, the arrows indicate bilateral ranulas (GB). B) Enlarged histopathological preparation of the sublingual salivary gland in patient 3 
ical evaluation of the aspirate is also of great diagnostic value $[17,18]$. In our cases, because of young age, biopsy would have to be performed with anaesthesia to make it safe for patients. In order not to expose the patients to unnecessary loads, it is better to do one intervention and examine collected tissues. Steelman et al. suggested monitoring in neonates with ranulae, who present with no eating or respiratory disorders [19]. Small and asymptomatic congenital ranulae may resolve spontaneously over time. However, monitoring is needed in all cases [20]. Seo et al. proposed maximum observation period not longer than three months [18]. However, there was an increase in the size of ranula leading to feeding difficulties in all our patients. The organs responsible for food intake, i.e. the lips, tongue, cheeks, palate will not create a closed space with low pressure, and visceral swallowing will be disturbed. Ineffective sucking causes milk spitting, which quickly alerts the parents due to a lack of weight gain and apathy of the child. Therefore, a decision was made to perform a conventional total excision of the sublingual gland. Histopathology showed distended salivary ducts in all cases.

Total excision of the gland along with the pseudocyst is the most common treatment approach described in literature. This method is predictable in most cases and is practically limited to the salivary gland [8]. Surgical excision should be performed carefully so as not to damage the nearby anatomical structures, i.e. submandibular gland duct and the lingual nerve. Recurrence rates are estimated at $1 \%$ and are usually seen in the case of salivary gland excision without removing the pseudocyst [21]. Marsupialisation is a less invasive method commonly used for the treatment of congenital ranula. It was reported that two cases of congenital ranula were successfully treated with marsupialisation at the age of 9 and 12 months [22]. However, the method is not always effective. Mneimneh et al. described a case of a fouryear-old girl with congenital ranula initially treated with marsupialisation. Unfortunately, the pseudocyst recurred after one week, and it was only after surgical excision that the ranula was finally eliminated [9].

There are a variety of methods used to treat ranulas: surgical excision of the ranula alone or with the entire salivary gland, marsupialisation, needle aspiration, sclerotherapy, cryotherapy, and laser excision. Since there are no international standards for the treatment of this type of lesion, the method of treatment is chosen by the attending physician [23]. Non-surgical therapeutic approaches include conservative treatment using nickel gluconate, mercurius heel and glandula submandibularis suis D10/D30/D200, and the OK - 432 therapy, which are interestingly classified as homeopathic remedies although they are more efficacious than marsupialisation $[13,17$, $24,25]$. These methods are used in older children and adults. Other authors showed that conservative treatment in a group of 17 children aged between 3 and 12 years allowed for complete recurrence-free recovery [17].
Patient exposure to general anaesthesia and postoperative complications were avoided, and the treatment costs were reduced [17]. Sclerotherapy using different substances is another common method. According to Brannan et al., the use of $98 \%$ ethanol and 3\% sodium tetradecyl sulphate (STS) with drainage was successful in $87.5 \%$ of cases. Three patients (12.5\%) had to undergo surgical treatment [26]. Wada described successful treatment using OK-432 in an infant with congenital ranula [24]. Use of OK-432, a substance prepared by incubating the culture of the low-virulence Streptococcus pyogenes treated with penicillin G, causes an inflammation inside the pseudocyst, absorption of fluid, and disappearance of the ranula [24]. In 2008, an innovative method by using botulinum toxin type A was described for treating ranula [27]. Ten years of studies comparing ranula treatment by aspiration, marsupialisation, and excision in neonates and infants have shown recurrence of within one year in 8 out of 11 children after the initial treatment with aspiration and marsupialisation. The final treatment was surgical salivary gland removal [28].

It has been reported by many authors that the possible complications after the treatment of ranula may include recurrence, oedema, injury to the submandibular salivary duct, arterial damage and bleeding, lingual nerve palsy (usually transient), and infection [25, 28, 29]. Postoperative complications in the form of swollen floor of the mouth and the pharyngeal region were observed in only one patient (GB). We expected elimination of the factor responsible for feeding difficulties and reduced weight gain. Improved weight gain and continued development were achieved in two children. Unfortunately, feeding problems in the third child (GB) were caused by other factors. The boy was diagnosed with Nijmengen breakage syndrome, which is characterised by microcephaly at birth, dysmorphic facial features, mild growth retardation, combined immunodeficiency, and predisposition to malignancies. Defective immunological status was also the probable reason for healing problems observed after surgical excision of congenital ranula [30]. All three of our patients had congenital defects. Congenital jaundice, congenital malformation of the fingers of both hands (oligodactyly) and facial dysmorphism were found in patient 1 . Tuberous sclerosis complex, which is a rare genetic neurocutaneous multisystem disorder with variable clinical phenotype, was detected in patient 2 [31]. It is believed that congenital ranula develops independently of systemic diseases or congenital defects. However, very few authors report the general condition of their paediatric patients [22]. This indicates the need for further studies on congenital ranula in the context of general heath.

\section{CONCLUSIONS}

Infants with congenital ranula preventing normal food intake require effective surgical treatment involving no risk of recurrence or the need for retreatment. 


\section{DISCLOSURE}

The authors declare no conflict of interest.

\section{REFERENCES}

1. Raju R, Digoy P. Management of pediatric ranula. Oper Tech Otolaryngol Head Neck Surg 2009; 20: 260-262.

2. Myers EN, Ferris RL. Salivary gland disorders. Springer 2007: 222234.

3. de Visscher JG, van der Wal KG, de Vogel PL. The plunging ranula. Pathogenesis, diagnosis and management. J Craniomaxillofac Surg 1989; 17: 182-185.

4. Mun SJ, Choi HG, Kim H, et al. Ductal variation of the sublingual gland: a predisposing factor for ranula formation. Head Neck 2014; 36: 540-544.

5. Zhang B, Yang Z, Zhang RM, et al. Are the patients with anatomic variation of the sublingual/Wharton's duct system predisposed to ranula formation. Int J Pediatr Otorhinolaryngol 2016; 83: 69-73.

6. Hze-Khoong EP, Xu L, Shen S, et al. Submandibular gland mucocele associated with a mixed ranula. Oral Surg Oral Med Pathol Oral Radiol 2012; 113: e6-e9.

7. McGurk M, Eyeson J, Thomas B, et al. Conservative treatment of oral ranula by excision with minimal excision of the sublingual gland. Histological support for traumatic etiology. J Oral Maxillofac Surg 2008; 66: 2050-2057.

8. McGurk M. Management of the ranula. J Oral Maxillofac Surg 2007; 65: 115-116.

9. Mneimneh S, Barazi R, Rajab M. A rare case of congenital ranula in an infant. Case Rep Otolaryngol 2016; 2016: 5874595.

10. Samour PQ, King K. Handbook of pediatric nutrition. Jones and Bartlett Publishers, Sudbury 2005: 75-106.

11. Flaitz CM, Hicks JM; Mucocele and Ranula. eMedicine 2015. Available from: http://emedicine.medscape.com/article/1076717-clinical

12. Molero-Osorio A, Santos-Bolivar J, Mencias O, et al. Early prenatal diagnosis of congenital ranula. Am J Sonogr 2019; 2: 7.

13. Haberal I, Göcmen H, Samim E. Surgical management of pediatric ranula. Int J Pediatr Otorhinolaryngol 2004; 68: 161-163.

14. Karam O, La Scala G, Extermann P, et al. Congenital sublingual cyst. Swiss Society of Neonatology 2005: 1-16.

15. Reddy A, Kreicher KL, Patel NA, et al. Pediatric epidermoid cysts masquerading as ranulas: A case series. Int J Pediatr Otorhinolaryngol 2016; 81: 26-28.

16. Baker HL. The application of magnetic resonance imaging in otolaryngology. Laryngoscope 1986; 96: 19-26.

17. Garofalo S, Mussa A, Mostert M, et al. Successful medical treatment for ranula in children. Oral Surg Oral Med Oral Pathol Oral Radiol 2014; 117: 289-297.

18. Seo JK, Park JJ, Kim HY, et al. Surgical management of intraoral ranulas in children: An analysis of 17 pediatric cases. Int J Pediatr Otorhinolaryngol 2010; 74: 202-205.

19. Steelman R, Weisse M, Ramadan H. Congenital ranula. Clin Pediat 1998; 37: 205-206.

20. Bernhard MK, Huckel D, Halama D. Congenital ranula in newborn. HNO 2007; 55: 388-391.

21. Zhao Y-F, Jia J, Jia Y. Complcations associated with surgical management of ranulas. J Oral Maxillofac Surg 2005; 63: 51-54.

22. Ghani NA, Ahmad R, Rahman RA, et al. A retrospective study of ranula in two centers in Malaysia. J Maxillofac Oral Surg 2009; 8: 316-319.

23. Zhi K, Gao I, Ren W. What is new in management of pediatric ranula? Curr Opin Otolaryngol Head Neck Surg 2014; 22: 525-529.
24. Wada T, Yamamoto H, Ootsuki R, et al. Congenital ranula. Asian J Oral Maxillofac Surg 2004; 16: 63-66.

25. Özkan A, Ulucanli S, Yaman H, et al. A case of congenital ranula operated in the early infancy. Eur J Basic Med Sci 2013; 3: 66-69.

26. Brannan ZJ, Lubeley LJ, Sutphen SA, et al. Percutaneous treatment of ranulas: ultrasound-guided drainage with salivary gland chemical ablation. Pediatr Radiol 2019; 49: 801-807.

27. Chow TL, Chan SWW, Lam SH. Ranula successfully treated by botulinum toxin type A: report of 3 cases. Oral Surg Oral Med Pathol Oral Radiol Endod 2008; 105: 41-42.

28. Zhi K, Wen Y, Ren W, et al. Management of infant ranula. Inter J Pediat Otorhinolaryng 2008; 72: 823-826.

29. Abdul-Aziz D, Adil E. Ranula excision. Oper Tech Otolayngol Head Neck Surg 2015; 26: 21-27.

30. Gregorek H, Olczak-Kowalczyk D, Dembowska-Bagińska B, et al. Oral findings in patients with Nijmegen breakage syndrome: a preliminairy study. Oral Surg Oral Med Oral Pathol Oral Radiol Endod 2009; 108: 39-45.

31. Henske EP, Jozwiak S, Kingswood JC, et al. Tuberous sclerosis complex. Nat Rev Dis Primers 2016; 2: 16035. 
